\title{
Charge Distribution Model in Cubic Perovskite-type Compounds
}

\author{
R. E. Alonso and A. López-García \\ Departamento de Física, Facultad de Ciencias Exactas, Universidad Nacional de La Plata, CC. 67, \\ 1900 La Plata, República Argentina \\ Reprint requests to Prof. A. L.-G.; fax: +54-221-425-2006, e-mail: abeti@ venus.fisica.unlp.edu.ar
}

Z. Naturforsch. 55 a, 261-266 (2000); received August 25, 1999

Presented at the XVth International Symposium on Nuclear Quadrupole Interactions, Leipzig, Germany, July 25 - 30, 1999.

\begin{abstract}
A non-zero electric field gradient tensor, detected by probes that occupy sites with cubic point group symmetry, was observed in many $\mathrm{ABO}_{3}$ perovskite-type compounds. This breakdown of local cubic symmetry is commonly associated with the presence of oxygen vacancies around the probe. This effect in $\mathrm{BaTi}_{x} \mathrm{Hf}_{1-x} \mathrm{O}_{3}$ with $x=0.7,0.5,0.3,0.1,0.05$ and 0.01 is studied in this work. The cell parameters were obtained at laboratory temperature using XRD spectroscopy. The hyperfine parameters were measured at a ${ }^{181}$ Ta probe in the B site using Perturbed Angular Correlations (PAC) spectroscopy as a function of both temperature and composition. As a common trend, a static asymmetric and distributed quadrupolar interaction, strongly dependent on composition has been observed. The results, together with those corresponding to $1>x \geq 0.75$, are analyzed using the point-charge model in terms of polarized oxygen vacancies, different covalence of the Ti-O and Hf-O bonds with computational simulation for the lattice positions of cations and oxygen vacancies.
\end{abstract}

Key words: Perovskites; Ferroelectrics Materials; Defects; Polarized Oxygen Vacancies; Electric Field Gardient.

\section{Introduction}

Perovskite-type compounds $\mathrm{AA}^{\prime} \mathrm{BB}^{\prime} \mathrm{O}_{3}$ can contain a large number of oxygen vacancies [1] which depend strongly on the preparation method and the subsequent thermal treatment. Some macroscopic physical properties are closely related with such defects.

Microscopic studies of perovskites have been done through the determination of the electric field gradient tensor (EFG) at probes located at A and B sites. However, the presence of oxygen vacancies does not allow a direct interpretation of the hyperfine parameters because the contribution of the oxygen vacancies to the EFG is still unknown.

Symmetry considerations show that at the position of the B cation in the cubic phase of stoichiometric perovskites the EFG is zero. This brings about a straightforward relation between the EFG in a nonstoichiometric cubic phase at probes placed in B-sites and the distribution of oxygen vacancies. Therefore we have studied in this work the hyperfine interactions in the cubic phase of $\mathrm{BaTi}_{x} \mathrm{Hf}_{1-x} \mathrm{O}_{3}$ with various values of $x$. The experimental results were obtained by X-ray diffraction (XRD) and Perturbed Angular Correlation (PAC) spectroscopy. Once these data are obtained, computational calculations using the pointcharge model (PCM) are used to interpret the characteristics of the EFG measured.

\section{PAC Measurements}

The samples were irradiated with a flux $\left(10^{13} / \mathrm{s}\right.$ $\mathrm{cm}^{2}$ ) of thermal neutrons at $300 \mathrm{~K}$ in order to activate the ${ }^{181}$ Ta probe obtained from ${ }^{180} \mathrm{Hf}$.

In this section the results of measurements on cubic $\mathrm{BaTi}_{x} \mathrm{Hf}_{1-x} \mathrm{O}_{3}$ with $x=0.7,0.5,0.3,0.1,0.05$; and 0.01 as a function of temperature are reported. These data will be added to previous ones obtained for $x>0.75$ [2].

In all samples the perturbation factors measured were fitted with a static, asymmetric and disordered 


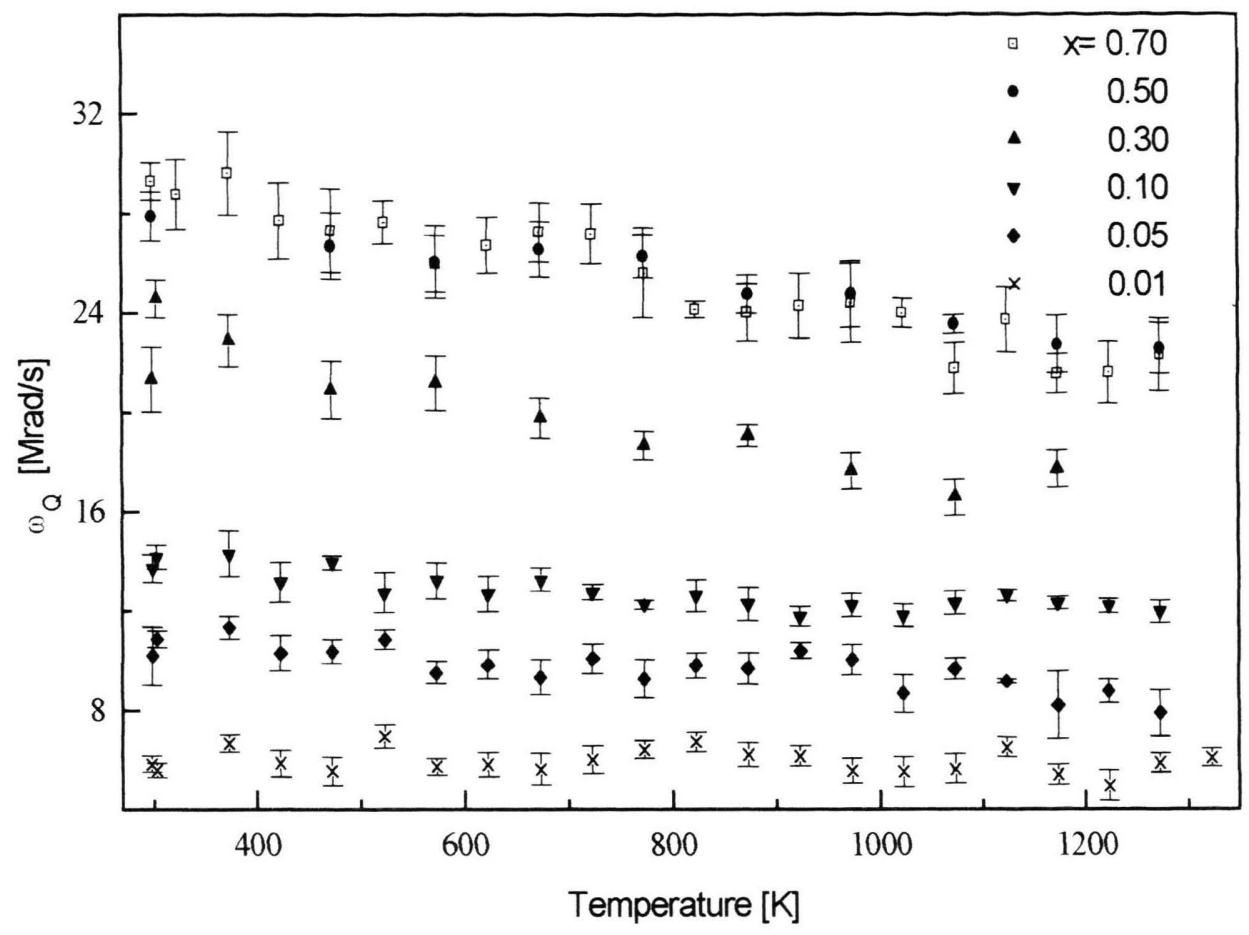

Fig. 1. Quadrupolar frequency as a function of temperature for the compositions measured.

electric quadrupole interaction. Figure 1 shows the fitted quadrupolar frequencies as functions of temperature and composition. The decrease of these with temperature is commonly associated with cell expansion. The asymmetry parameter (mean value $\eta=0.42$ ) is almost independent of temperature and composition. The distribution width $\delta$ is large and independent of temperature: for $x=0.7,0.5,0.3,0.1,0.05$ and 0.01 resulted $\delta=32,35,35,38,40$, and $75 \%$, respectively.

The existence of a finite quadrupolar frequency at all measured compositions is not compatible with the cubic symmetry of the B- or the A-site. On the other hand, ${ }^{181} \mathrm{Ta}$ can occupy neither an $\mathrm{O}$ nor an interstitial site.

Figure 2 shows the dependence of $\omega_{\mathrm{Q}}, \eta$ and $\delta$ on the composition, obtained with values fitted or extrapolated (for $x>0.75$ ) to room temperature. The quadrupolar frequency $\omega_{\mathrm{Q}}$ has a maximum for $x \approx 0.7$. At a first glance, this behavior of $\omega_{\mathrm{Q}}$ cannot be explained only in terms of oxygen vacancies because all samples were prepared in the same way, i.e., as a first approximation it can be expected that all samples had the same oxygen vacancy content.

\section{EFG Produced by Oxygen Vacancies}

The Point Charge Model (PCM) of the EFG gives results that are only usable in a comparative sense between similar samples. Although in general the calculated EFG tensor does not agree with the experimental one, the relative trends of $\omega_{\mathrm{Q}}$ and $\eta$, when varying some parameter, are of interest. On the other hand, the PCM is a simple method that can deal in a straightforward way with disordered systems with many atoms (2000 and more). For dealing with such systems with ab-initio molecular dynamics or Monte Carlo methods the computational work must be enormous.

As mentioned previously, the solid state reaction technique used for sample preparation can generate distinct concentrations of oxygen vacancies. The amount of these defects is expected to be greater than $2 \%$ in perovskites prepared by this method. Such vacancies produce long range fields.

A low EFG would indicate that the oxygen vacancies are randomly distributed and are not attracted by the ${ }^{181} \mathrm{Ta}$ PAC probe. 


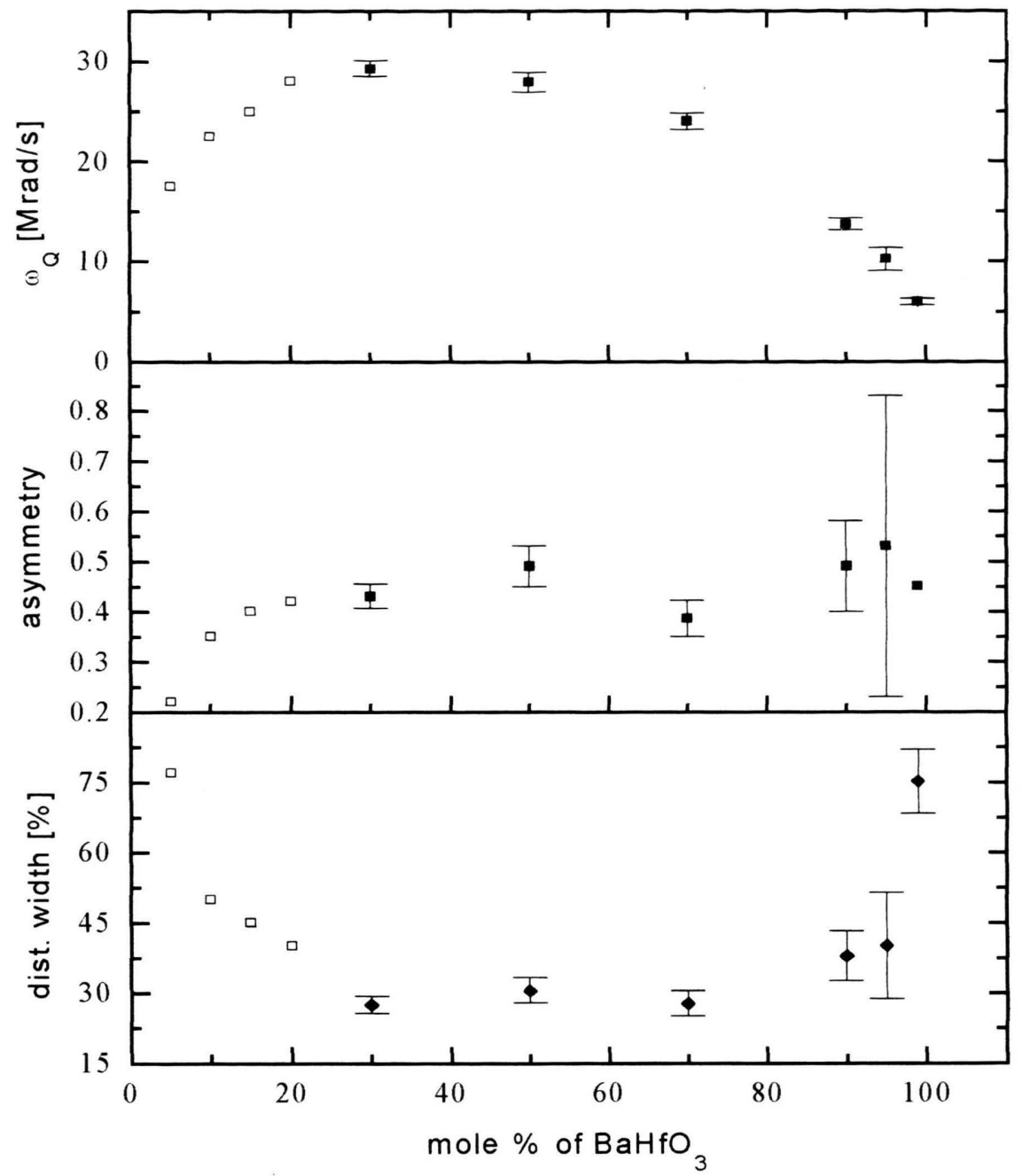

Fig. 2. The hyperfine parameters vs. $x$. The open squares correspond to room temperature extrapolated values (See text).

The calculation of the EFG was performed on samples of $10 \times 10 \times 10$ unit cells. The calculation was done by dividing the lattice into shells. The central shell is a cube with Ta at the center, 6 O's (2-) at the center of the faces and $8 \mathrm{Ba}$ 's $(2+)$ at the corners. The first shell consists of 26 cubes surrounding the central one, that have $\mathrm{Ti}(4+)$ or $\mathrm{Hf}(4+)$ at B sites randomly distributed according to the value of $x$. The second shell consists of 98 unit cubes covering the first one, and so on. Then, for a given concentration of oxygen vacancies, random vacancy positions were generated. The as produced configuration represents a possible neighborhood at each PAC probe, and the EFG tensor was calculated. This process was repeated 50000 times to generate a corresponding histogram. The cell parameter in the cubic structure was $a=1$, so the results for $V_{z z}$ are scaled by a factor $1 / 4 \pi \varepsilon_{0} a^{3}$.

One way to take into account the ${ }^{181}$ Ta-oxygen vacancy interaction is by changing the charge of the vacancies around the probe. The oxygen vacancies are created during the synthesis process. The mean life of the ${ }^{181} \mathrm{Ta}$ is too short to enable anion diffusion during the life time of the metastable state. Two electrons appear for each oxygen vacancy. If these electrons are trapped by a vacancy, the oxygen octahedra will have almost cubic symmetry and no EFG would be produced. On the other hand, if one or no electron is trapped, EFG's can be created. Due to the great mobility of the electrons they may rearrange in order to minimize the total energy. Thus, the original random distribution of oxygen vacancies has charge 


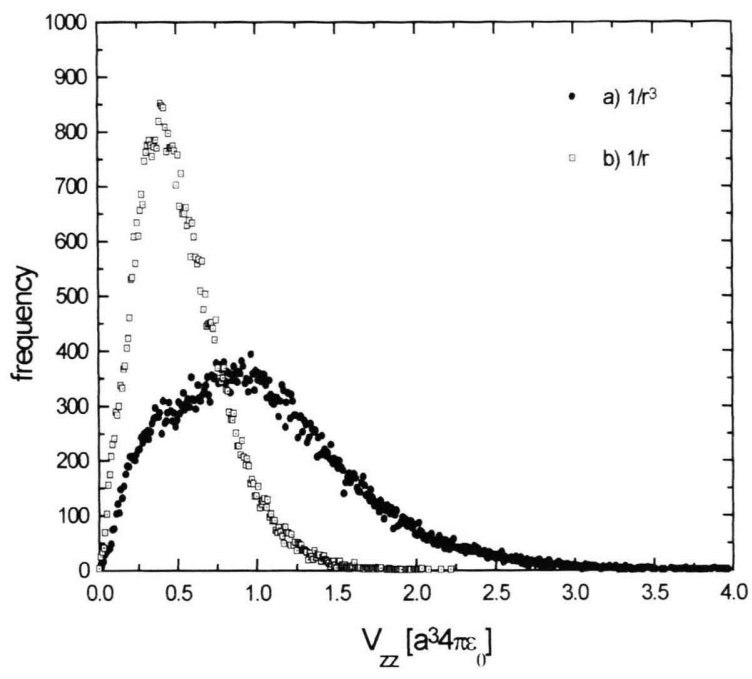

Fig. 3. $V_{z z}$ distribution functions for a sample with $3 \%$ oxygen vacancy content and polarisation profiles of type a) $1 / r^{3}$, b) $1 / r$. The frequency is the number of times that a calculated value of $V_{z z}$ is obtained.

states that depend on the distance to the Ta probe. The boundary conditions taken here are $q=-2$ e for vacancies in the octahedron surrounding the probe and $q=0$ for vacancies far away from this octahedron.

In Fig. 3 the $V_{z z}$ distribution functions are shown for $3 \%$ randomly distributed oxygen vacancies with electric charges modulated by the functions $1 / r$ and $1 / r^{3}$ and with the above boundary conditions. Both curves show that the peaks corresponding to vacancies present in the first, second and third shells overlap, forming an asymmetric and broad unique peak. The figure shows the different profiles of the distribution that can be obtained with different polarizing functions. The distribution width for a) and b) is nearly $100 \%$ and greater than $200 \%$, respectively. Broad distributions have been observed experimentally in several perovskites [3 - 5]. This simple picture gives a reasonable explanation of their origin.

In order to find the curve that reproduces the experimental results, several parameters must be determined (the precise amount of oxygen vacancies, the actual function that describes the charge distribution of oxygen vacancies, etc.). The interesting issue is that with a very simple hypothesis two observed experimental results can be explained: the presence of a non-zero EFG and the broad $V_{z z}$ distribution widths in sites with cubic symmetry in perovskites. From the above discussion it can be concluded that the origin of the $\mathrm{EFG}$ in $\mathrm{B}$-sites in perovskites is related to the presence

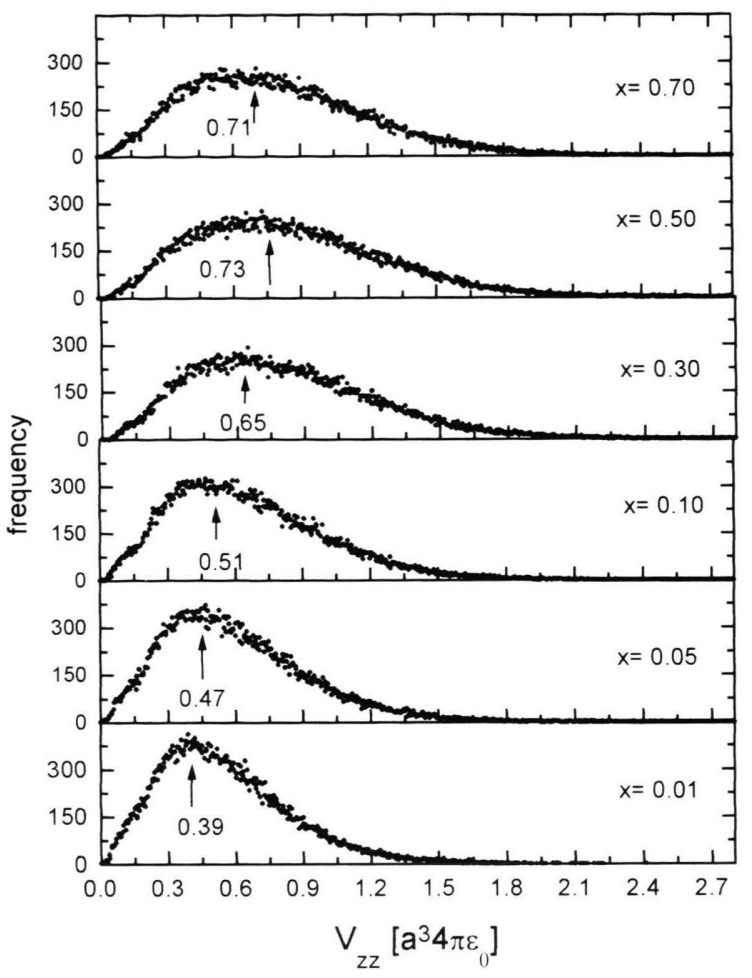

Fig. 4. $V_{z z}$ distribution for $\delta=0.3$ e and $3 \%$ oxygen vacancies for distinct values of $x$. The arrows indicate the position of the maximum.

of oxygen vacancies, and the distribution widths of $\omega_{\mathrm{Q}}$ observed are due to the charge polarization around the ${ }^{181}$ Ta probes (contributions to polarization of cations $\mathrm{A}$ and $\mathrm{B}$ were discarded for simplicity). The PAC spectra fitted with a unique distributed site (see PAC section) would mean that the probe is surrounded by several vacancies with different charge depending on their distance from the probe atom.

\section{Disorder Introduced by B-cation Substitutions}

The insulating perovskites $\mathrm{ABO}_{3}$ are known to be almost ionic compounds. However, in a previous theoretical work about the cubic phases of $\mathrm{SrTiO}_{3}$ and $\mathrm{SrHfO}_{3}$ it was shown that the covalence of oxygen $2 \mathrm{p}$ electrons is bigger for the Ti-3d electrons than for $\mathrm{Hf}$ $5 \mathrm{~d}$ electrons [6]. The difference ammounts to about $0.7 \mathrm{e}$, while the $\mathrm{Sr}$ ion has a purely ionic electronic shell structure. According to this hypothesis, in the present case, where there is $\mathrm{Ba}$ in place of $\mathrm{Sr}$ in the A-site, the Ti-O bond would be more covalent than the Hf-O bound. 
In the PCM it is normally assumed that the charges are concentrated at the ion's site with a charge equal to its nominal valence. But if the compound is covalent, the charge is distributed in space and the PCM is not applicable. Thus, for simulating the partial covalence of a bond we assume as a first approximation that the ionic charge is reduced from its nominal value. In order to study the variation of the EFG with the molar percentage of $\mathrm{BaHfO}_{3}$, the Ti ions were charged with $+4 \mathrm{e}-\delta$ and the $\mathrm{Hf}$ ions with $+4 \mathrm{e}$.

If $\mathrm{Ti}$ and $\mathrm{Hf}$ covalence is added to the EFG produced by polarized oxygen vacancies, the $V_{z z}$ distribution function changes drastically. In Fig. 4 the results obtained for $\delta=0.3 \mathrm{e}, 3 \%$ oxygen vacancies content and a charge polarization of the type $1 / r$ for different Ti:Hf ratios are shown. The $V_{z z}$ distribution curves now have one broad peak whose maximum position depends on composition as is observed experimentally.

The asymmetry parameter $\eta$ measured (see Fig. 2) is $\approx 0.45$ for $x=0.7,0.5,0.3$ and 0.1 and tending to zero as $x \rightarrow 1$. In samples with $x=0.05$ and 0.01 the fitted $\eta$ 's have big relative errors. This model predicts a mean asymmetry parameter of about 0.5 , independent of Ti:Hf ratio.

In this family of compounds the measured relative width depends strongly on the Ti-Hf composition. For the richest $\mathrm{Ti}$ or Hf compositions $\delta$ increases up to $\approx 80 \%$, while for middle compositions $\delta \approx 30 \%$ and almost constant. The values predicted by the model do not reproduce this fact because $\delta$ is maximum for $x=0.5$ and diminishes for $x$ close to 0 and 1 . This is expected. As was shown in Fig. 3 and the related discussion, the width of the EFG distribution function is strongly dependent on the profile selected for the polarisation of oxygen vacancies. Moreover, the tests performed on changing the difference of charge between $\mathrm{Hf}$ and $\mathrm{Ti}$ by distinct values $(0.2 \mathrm{e}, 0.4 \mathrm{e}$, etc.) show that again the width of the distribution varies with this parameter. In the real compound it is expected that the polarisation profile and the grade of covalence depend on the local disorder. Thus, the experimental distribution width dependence on composition can be reproduced by adjusting these two quantities.

From the maximum values of the $V_{z z}$ distribution functions shown in Fig. 4 and the lattice constants measured by XRD, the values of $V_{z z}=4 \pi \varepsilon_{0} a^{3} V_{z z}$

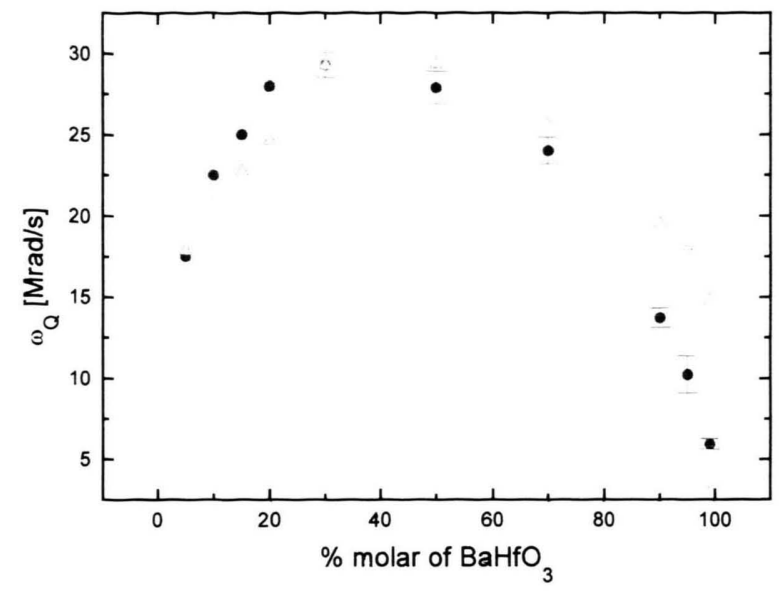

Fig. 5. Comparison between experimental (circles) and calculated (triangles) data. The calculated data have been normalised for coinciding both values at $x=0.70$.

can be calculated and compared with the experimental results. In Fig. 5 the measured and calculated $V_{z z}$ values are shown. Taking into account the simplicity of the model and the hypothesis used, the agreement is reasonable. Thus, it can be concluded that the EFGs measured in the $\mathrm{BaTi}_{x} \mathrm{Hf}_{1-x} \mathrm{O}_{3}$ family depend mainly on polarized, randomly distributed oxygen vacancies and on the different covalence of the B-O bonds.

\section{Conclusions}

For several compositions of the compounds $\mathrm{BaTi}_{x} \mathrm{Hf}_{1-x} \mathrm{O}_{3}$ the lattice constant at room temperature and the hyperfine interactions at the B-sites as functions of the temperature were measured. Above room temperature all the crystals were cubic. In each sample a non-zero, asymmetric and disordered EFG was obtained. This fact was previously observed in other perovskite-type compounds. The EFG at room temperature depends strongly on $x$. Using some simple assumptions and the PCM, we conclude that EFG's are related with the presence of oxygen vacancies. The behavior of the distribution width can be explained by the polarization of the oxygen vacancy charge as a function of the distance from the PAC probe atom. The dependence of the EFG on the mole percentage of $\mathrm{BaHfO}_{3}$ can be reproduced taking into account the different degree of covalence of the Ti-O and $\mathrm{Hf}-\mathrm{O}$ bonds. 
[1] M. A. Bunin, S. A. Prosandeyev, I. I. Gegusin, and I. M. Tennenboum, Rad. Eff. Def. Solids 134, 75 (1996). M. V. Raymond and D. M. Smyth, J. Phys Chem. Solids 57, 1507 (1996). W. L Warren, K. Vanheusden, D. Dimos, G. E. Pike, and B. A. Tuttle, J. Amer. Ceram. Soc. 79, 536 (1996).

[2] A. Ayala, PhD Thesis, Universidad de La Plata, 1996.

[3] R. E. Alonso, P. de la Presa, A. Ayala, and A. López
García, J. Physics: Cond. Mat. 10, 2139 (1998).

[4] G. Catchen, S. Wokitch, D. Spaar, and M. Blaszkiewicz, Phys. Rev. B42, 1885 (1990).

[5] G. Catchen, E. Hollinger, and T. Rearick, Z. Naturforsch. 51a, 411 (1996).

[6] G. Fabricius, E. L. Peltzer y Blancá, C. O. Rodríguez, A. P. Ayala, P. de la Presa, and A. López García, Phys. Rev. B55, 41 (1997). 